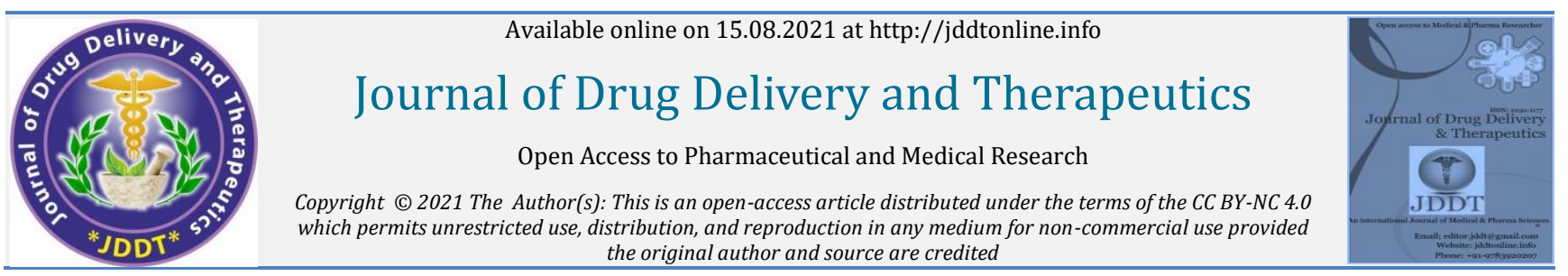

Open Access Full Text Article the original author and source are credited

Review Article

\title{
Niosomes as Nano-carrier Based Targeted Drug Delivery System
}

\author{
Chanchal Joy, Smitha K. Nair*, Krishna Kumar K, Dineshkumar B, \\ Dept. of Pharmaceutics, St. James' College of Pharmaceutical Sciences, Chalakudy, Kerala \\ St. James' Hospital Trust Pharmaceutical Research Centre (DSIR Recognized), Chalakudy
}

\begin{tabular}{ll}
\hline Article Info: & \\
\hline Cite this article as: & Article History: \\
\hline
\end{tabular}

Joy C, Nair SK, Krishna Kumar K, Dineshkumar B, Niosomes as Nano-carrier Based Targeted Drug

Delivery System, Journal of Drug Delivery and

Therapeutics. 2021; 11(4-S):166-70

DOI: http://dx.doi.org/10.22270/jddt.v11i4-S.4907

\section{Abstract}

Niosomes are considered as novel nano-carrier in the drug delivery systems. These are much more stable than other nano-carriers like liposomes and nanoparticles. It is an example for targeted and control release of medication. The drug is entrapped into lipid core so that it prevents the drug from leaching. Here the drug will be targeted only to the desired cell/tissue and not to the non-targeted cells. By giving this formulation we can thereby reduce the dose of the drug and toxicity. This review article mainly emphasizes on the potential applications of niosomes in targeted drug delivery system.

Keywords: niosomes, nanocarriers, targeted drug delivery.

*Address for Correspondence:

Smitha K. Nair, Dept. of Pharmaceutics, St. James' College of Pharmaceutical Sciences, Chalakudy, Kerala

\section{INTRODUCTION}

Niosomes are formation of vesicles prepared by the mixing of cholesterol and a non-ionic surfactant. It is an example for targeted and controlled release of medicament. There are various approaches for delivering these drugs like liposomes, niosomes, nanoparticles etc. Niosomes are preferred over liposomes because the former exhibit much more stability and economic. They enhance the therapeutic efficacy of the drug molecules by delaying the clearance from the circulation and protecting from environment. Niosomes are widely used for targeted drug delivery system ${ }^{1}$. Paul Ehrlich in 1990 started the era of targeted drug delivery systems. The major mechanism is that the drug will be targeted only to its site of action and not to the non-targeted cells. Hereby we can reduce the toxicity. The formulation of niosomes consists of non-ionic surfactants eg. span 60 along with cholesterol is added in order to attain the stability of formulation.

\section{ADVANTAGES}

- The characteristics features like size, shape, nature can be changed.

- Enhances the stability of the entrapped drug.

- Improves the penetration of drug into the skin.

- They are osmotically active hence has longer storing period.

- The vesicles act as a transporter for the slow release of medication in a controlled manner.

- Better patient compliance 2 .

\section{DISADVANTAGES}

- Leaching of drug may occur.

- Difficult method of preparation.

- Aggregation or fusion.

- Stability issues.

\section{STRUCTURE OF NIOSOMES:}

It includes 3 major components:

1. Non-ionic surfactants.

2. Cholesterol.

3. Charge inducing molecule ${ }^{3}$.

Non-ionic surfactant: These have no charged groups in their structure. These are much more stable, biocompatible and less toxic than anionic, amphoteric and cationic surfactans. Non-ionic surfactants are amphiphilic molecules that include two different regions: one of them is hydrophilic (water-soluble) and the other one is hydrophobic (organic soluble).

Eg: spans 20,40,60 tween 20,40,80

Cholesterol: Cholesterol helps in the formation of hydrogen bonds with hydrophilic head of surfactant. The amount of cholesterol in the formulation influences the stability of the niosome. Cholesterol enhances the rigidity of the vesicles. 
Drug entrapment efficiency can be altered by using various concentration of cholesterol.

Charge inducing molecule: The presence of a charge inducing molecule enhances the stability of the niosome formulation. Diacetyl phosphate and phosphatidic acid are commonly used to induce negative charge to the niosome formulation similarly stearylamine is used to induce positive charge to the formulation. The concentration must be 2.5-5 mol\%.

\section{CLASSIFICATION OF NIOSOMES:}

a) Small unilamellar vesicle $0.025-0.05 \mu \mathrm{m}$.

b) Large unilamellar vesicle $\geq 0.05 \mu \mathrm{m}$.

c) Multi lamellar vesicle $\geq 0.10 \mu \mathrm{m}^{4}$.

\section{METHOD OF PREPARATION:}

General Method of Preparation 5:

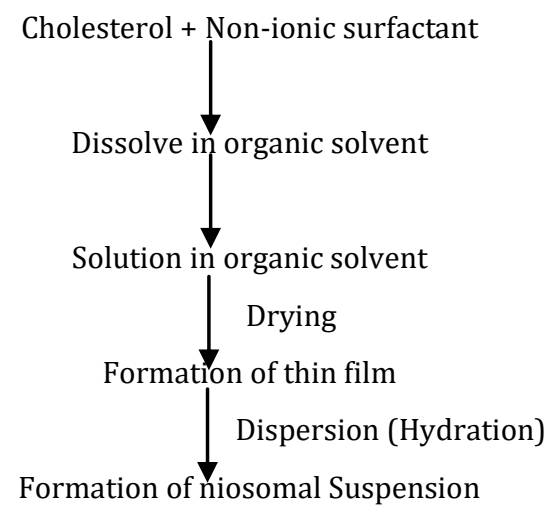

\section{Thin Film Hydration Technique (Hand shaking method)}

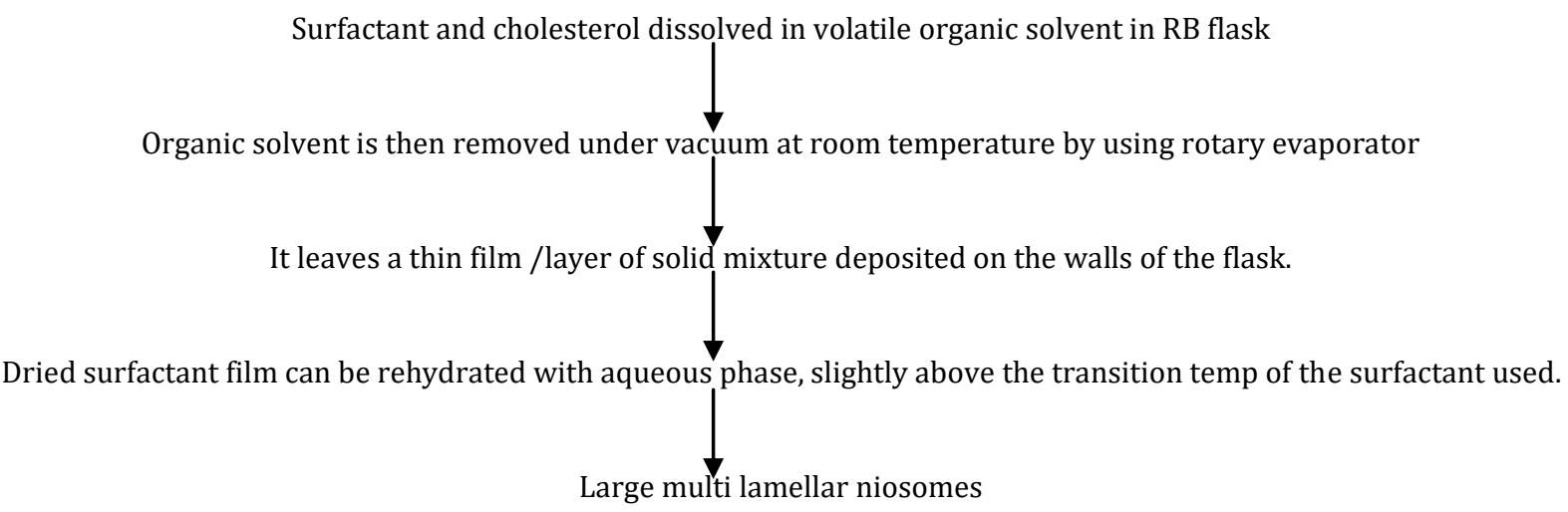

2. Ethanol Injection Method:

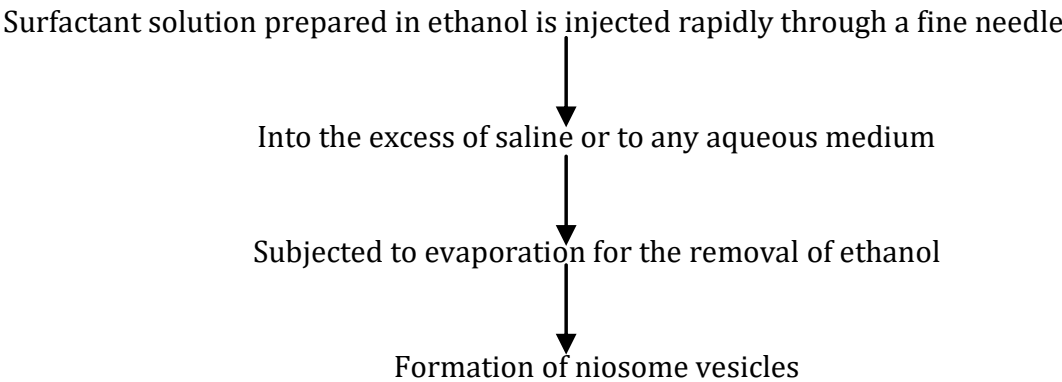

Various other methods can also be used for the Preparation of niosomes:

- Sonication method

- Multiple Membrane extrusion method.

- Micro fluidization

- Reverse phase evaporation

- Bubble method

- Tran's membrane pH gradient Drug Uptake Process method

\section{APPLICATION OF NIOSOMES}

Niosomes as drug delivery systems: The fundamental purpose of a targeted drug delivery system is to increase the selectivity towards the targeted tissue and also to reduce the toxicity of the medication. In 1909 Paul Ehrlich developed a drug delivery system that can directly target the drug to a diseased cells and tissues. Various systems such as Immunoglobulins, liposomes, microspheres and Niosomes 
were used as a vehicle for the drug to the target cells and tissues. Among these, liposomes and niosomes obtained special consideration due its unique properties and stability features. Niosomes have the ability to entrap both hydrophilic and lipophilic drugs. Presence of non-ionic surfactant and the lipid makes niosomes a better carrier of $\operatorname{drug}(\mathrm{s})$ to tumor, liver and brain. They are widely used nowadays in targeting of the drug for treating cancers, parasitic, viral and other microbial diseases more effectively 6.

Aerosolized Niosomes with anti-cancer potential: Gemcitabine and cisplatin loaded aerosolized niosomes was synthesized for the treatment of lung cancer. Cytotoxicity study of formulation against normal lung (MRC5) and lung cancer (A549) cell lines was investigated. The results revealed that the optimized formulation had reduced cytotoxicity against both MRC5 and A549 cell lines when compared with the control system (Gem + Cis alone) from highly toxic (IC50 $<1.56 \mu \mathrm{g} / \mathrm{mL}$ ) to weakly toxic (IC50 $280.00 \mu \mathrm{g} / \mathrm{mL}$ ) and fairly toxic (IC50 $=46.00 \mu \mathrm{g} / \mathrm{mL}$ ), respectively, after $72 \mathrm{~h}$ of treatment. These findings promised that the optimized aerosolized niosomal formulation has excellent anti-tumor activity and can be used as promising drug delivery system ${ }^{7}$.

Niosomes improved the bioavailability of atorvastatin calcium: Atorvastatin calcium is an $\mathrm{HMG}-\mathrm{CoA}$ reductase inhibitor. The oral bioavailability is less than $12 \%$. The drug is highly soluble in acidic $\mathrm{pH}$ and is absorbed in upper GIT. Therefore, to compensate these problems atorvastatin calcium has been formulated in the form of niosomes. The niosomes were prepared using thin film hydration technique using different grades of surfactants like span 40, span 60 and span 80 . The characterization studies were performed and evaluvated for its shape, size and entrapment efficiency. The study proved that atorvastatin calcium niosomes has improved the oral bioavailability and stability of the formulation proving to be one of the promising and fundamental nanocarrier. ${ }^{8}$.

Methylene Blue loaded niosomes with wound healing: Methylene Blue (MB) loaded niosomes were prepared by using the ultra-sonication method as a green formulation method. The prepared niosomal formulation were optimized by using Behnken design. Characterization studies were performed to evaluvate the vesicle shape, drug entrapment efficiency and zeta potential. The drug entrapment efficiency of the formulation was increased. Male Wistar rat was used to study the In vivo efficiency of enhanced formulation. The levels of Superoxide dismutase (SOD) and malondialdehyde in the skin sample were evaluated which can cause tissue damage and delay in wound healing ${ }^{9}$.

Levofloxacin loaded niosomes with anti-bacterial: Levofloxacin entrapped niosomes were prepared and examined the effectiveness in treating Pseudomonas aeruginosa infections in Dawlwy rats. Thin film hydration method is used to develop the niosomes and then niosomes was assessed for in vitro drug release and stability. Studies were conducted by using drug free niosomes, Drug loaded niosomes and free drug and blood samples were collected. The results revealed that the niosome treated group significantly reduced complete blood counts and viable bacterial counts (CFU) in the liver, kidney, and spleen ${ }^{10}$.

D-limonene-loaded niosomes with cytotoxic activity: Dlimonene-loaded niosomes (D-limonene/Nio) was developed for tumor therapy. The niosomal formulation was synthesized by conventional thin film hydration technique with Span ${ }^{\circledR}$ 40: Tween ${ }^{\circledR}$ 40: cholesterol (35:35:30 molar ratio) and characterized. The entrapment efficiency of the formulation was found to be about $87 \pm 1.8 \%$. Prolonged release of $\mathrm{D}$-limonene was observed from prepared niosomes when compared to free D-limonene. The cytotoxicity study results revealed that the prepared niosomes increase the cytotoxic activity with all three cancer cell lines (HepG2, Macf-7 and A549) at the concentration of $20 \mu \mathrm{M}^{11}$

Mirtazapine loaded niosomes - improved bioavailability: mirtazapine loaded niosomes was synthesized for nasal drug delivery. Mirtazapine is an anti-depressant drug with poor oral bioavailability. Therefore, the drug has been formulated in the form of niosomes mainly to improve the bioavailability. Niosomes was formulated by using thin film hydration technique and optimized using $3^{2}$ full factorial design. Mirtazapine loaded niosomes was then fused into a Poloxamer 407 and Carbopol 934 in-situ gel. The study revealed that niosomal in-situ gel of Mirtazapine can be used for a prolonged period by minimizing drug administration frequency and thus improves the bioavailability ${ }^{12}$.

Quercetin loaded niosomes - improved solubility: flavanoid loaded niosomes was prepared to improve its solubility, stability and penetration ability. Whitening and antioxidant Properties of several flavonoids were studied, including quercetin, morin, myricetin, rutin, and breviscapine. The drug loaded niosomes were characterized and entrapment efficiency was determined. The photostability, solubility, release behavior, ex vivo drug penetration, and skin retention properties of Niosome preparation were studied. Drug entrapment efficiency of quercetin loaded niosomes was found to be high. The noisome preparation remarkably enhanced the solubility and photo-stability of drug quercetin 13 .

Temozolomide loaded niosomes - Brain drug delivery: Temozolomide incorporated niosomes was used for the treatment of glioblastoma, a destructive brain tumor. The prepared niosomes are then surface modified with chlorotoxin, which is a small 36 amino acid peptide. It will produce an increase in the accumulation of drugs in the cerebri of brain by active targeting mechanism. Temozolomide-loaded niosomes were developed using thinfilm hydration method and then characterized. Quantitative tissue distribution studies revealed that enhanced permeation of the medication into the brain was achieved by the noisome formulation 14 .

Glipizide-loaded Niosomes - improved bioavailability: Glipizide-loaded Niosomes was formulated to enhance the oral bioavailabilty of the drug. Highest percentage of drug entrapment was observed in the optimization process. The in vitro drug release studies from Niosomes showed a prolonged drug release for more than $24 \mathrm{~h}$. Zeta potential value is found to be negative which reveals that Glipizide Niosomes were stabilized by electrostatic repulsive forces. Maximum entrapment and suitable drug release rate are observed for invitro evaluation of the Glipizide niosomes. Niosomal formulation of glipizide is found to improve the bioavilability and prolonged drug release profile 15 .

Carum loaded niosomes-anticancer activity: Carumloaded niosomes was prepared and evaluvated on breast cancer cells. Thymoquinone (TQ), a phytochemical compound found in carum seeds (C.carvil) was the drug used. In this study, they introduced an innovative noisome delivery system with ergosterol (herbal liquid) in structure, instead of cholesterol. It resulted in high stability, nanometric size, good encapsulation efficiency niosomes. In this study they evaluated properties of TQ, TQ loaded niosomes, carum and carum loaded niosomes and their efficiency was compared on the breast cancer cells ${ }^{16}$. 
Pegylated niosomal with albendazole - anti-fungal activity: Pegylated niosomal formulation of albendazole was developed for the treatment of echinococcosis. The main purpose of the study is to enhance the systemic residence time of the albendazole which would help in the treatment of echinococcosis. Niosomes was synthesized by using ethanol injection method and characterization studies were conducted to evaluvate morphological size, shape, and in vitro release study.the results concluded that the niosomal formulation have the ability to use in the treatment of echinococcosis and hydatid cysts. 17

Cisplatin loaded niosomes-anticancer activity against Breast cancer cells: In this study they prepared nanoniosomated cisplatin and evaluated its efficacy in the BT-20 cell line in vitro. Niosome containing cisplatin were synthesized by reverse-phase evaporation method and characterized. Further studies revealed that the niosomes increased the cytotoxic effects of cisplatin by 1.5 times than the standard drug. The study confirms that niosomes proves to be a promising nanocarrier for cisplastin. ${ }^{18}$.

Herbal drug loaded niosomes: Herbal drug loaded niosomes was prepared as topical gel for transdermal drug delivery. Niosomes were synthesized by using thin film hydration technique. The herbal drug was successfully loaded in the niosomes using span 20,60, 80 and cholesterol. The prepared niosomes were assessed for their particle size, morphology and in vitro drug release and the optimized formulation (batch 7) shows highest drug entrapment efficiency. Plain herbal gel and niosomal herbal gel were compared for their in vitro drug release properties and niosomal gel preparation gives better results 19 .

Curcumin loaded niosomes -anticancer activity against ovarian cancer cells: Curcumin loaded niosomes, its characterization and cytotoxic effect on ovarian cancer cells was studied. Curcumin is a poorly water-soluble drug with rapid degradation. Hence it has been formulated in the form of niosomes inorder to compensate these disadvantages. Curcumin has been encapsulated into the noisome core using non-ionic surfactants like span 60 and tween 80. Entrapment efficiency was increased. Curcumin niosomes proved to improve the cytotoxic activity and apoptotic rate against ovarian cancer 20 .

Artemisone loaded niosomes- skin anticancer activity: Artemisone is a 10-amino-artemisinin moiety, having antimalarial property along with anticancer activity. In this study they developed artemisone loaded niosomes using thin-film hydration technique. Cytotoxicity study results shows high selectivity towards the tumor cells with inconsequential toxicity towards the normal skin cells. The artemisone loaded niosomes completely inhibited melanoma cells than the free artemisone drug 21.

Benzoyl peroxide loaded niosomal gel - Anti-bacterial activity: Benzoyl peroxide loaded niosomal gel was synthesized for its topical delivery in to the skin. The main aim of the study was to improve the drug permeation through the skin. Later the niosomes were further loaded in the carbopol gel to increase the contact time of the gel. The study revealed that the prepared niosomal gels have the ability to improve the drug permeation through the skin. After four days of treatment the niosomal gel reduced the bacterial infection. The study concluded that noisome formulation has got much more advantages than other conventional dosage forms 22 .

Diclofenac sodium loaded niosomes: Diclofenac sodium multilamellar vesicle (MLVs) was formulated using thin film hydration method. Cholesterol and surfactant (span 60) were used in different ratio for the preparation. In vitro drug release from the preparation was found to be consistent and a stability study was conducted to in order to learn the ability of drug to be leached out from the formulation upon storage at room temperature $25 \pm 20 \mathrm{C}$ as well as in refrigerated condition (20C to $80 \mathrm{C}$ ). The considerable stability of niosomal formulation was found to be 2 weeks. In short encapsulation of diclofenac sodium in the niosomal suspension was found to be increased when compared to the conventional drug ${ }^{23}$.

Tenofovir disoproxil fumarate loaded niosomes improved bioavailability: Tenofovir disoproxil fumarate (TDF)niosomes has been prepared with the aim of improving the oral bioavailability of which is an anti-HIV drug. Thin film hydration technique was used Different concentration of surfactant, cholesterol, and dicetyl phosphate were used to formulate the niosomes. Niosomes prepared with 1:1:0.1 ratios of surfactant: cholesterol: dicetylphosphate with each grade of span shows higher entrapment efficiencies. Maximum drug release $(99.091 \%)$ is obtained for the niosomes prepared with sorbitan monostearate with zero prder rate kinetics. In vivo study results revealed that the prepared niosomes significantly improves the oral bioavailability of TDF after the dose of $95 \mathrm{mg} / \mathrm{kg}$ in rats. By using TDF loaded niosome more than two-fold enhancement in the oral bioavailability of the drug is observed 24 .

Itraconazole loaded niosomes- anti-fungal activity: Itraconazole loaded niosomes was formulated and evaluated for anti-fungal activity. Factorial design is used to optimize the Surfactant: cholesterol ratio and quantity of ethanol used in the formulation was studied. Vesicle size, entrapment efficiency, drug release, skin permeation, and antimycotic activity of the prepared niosomes were studied. Further study results revealed that Itraconazole noisome has much more inhibitory action than the marketed formulation against $C$. albicans 25 .

Isoniazid loaded niosomes-anti-tuberculosis activity: Isoniazid loaded niosomes was synthesized by using reverse phase evaporation method, which was used for the treatment of tuberculosis. The surfactants used was span 60 and span 20. A charge inducing agent like di-acetyl phosphate was added. Characterization studies were conducted inorder to determine the zeta potential, particle size and poly dispersibility index.The study revealed that isoniazid formulations has reduced dose and toxicity when given parenterally or by any other routes 26 .

Cantharidin loaded niosomes-anticancer activity against breast cancer cells: Cantharidin loaded niosomes was synthesized by using injection method. The ability in intensifying the antineoplastic activities of the drug and decreasing its toxicity was studied on human breast cancer cell line MCF-7. The in vivo therapeutic efficacy of the preparation was examined in S180 tumor-bearing mice. $1.0 \mathrm{mg} / \mathrm{kg}$ niosomal-cantharidin formulation was administrated into mice, showed most effective antitumor activity, with an inhibition rate of $52.76 \%$. The activity of the formulation found to be significantly higher than that of concentration of free cantharidin 27

Tamoxifen citrate loaded niosomes-anticancer activity against breast cancer cells: Tamoxifen citrate loaded niosomes was synthesized by conventional thin film hydration technique for the treatment of human breast cancer. The niosomal formulation of tamoxifen was optimized and showed considerably increase in cellular uptake (2.8-fold) and exhibited appreciably larger anticancer activity on MCF-7 breast cancer cell line. Niosomal 
tamoxifen showed improved tumor volume reduction when compared to free tamoxifen ${ }^{28}$.

Doxorubicin loaded noisome-anticancer activity against breast cancer cells: An effective tumor-targeted noisome drug delivery system was intended and developed. Niosomes were synthesized by using a combination of surfactant (Pluronic L64) and cholesterol. The drug doxorubicin was loaded into the niosome, then transferrin was conjugated to niosomes surface. They have studied the anticancer activity against MCF-7 and MDA-MB-231 tumor cell lines. A markable decrease in viability is observed 29 .

\section{CONCLUSION}

Niosomes as nano-carrier can be used for targeted drug delivery system. niosome formulation has been reported as stable formulation and anti-cancer drugs, anti-bacterial drugs, anti-inflammatory drugs, anti-viral drugs can be encapsulated. Niosomes can be used as promising drug nanocarriers to achieve better bioavailability and targeting approaches. Thus, niosomes drug delivery requires further research on correlation of in vivo and in vitro study results. So, this will bring out the noisome formulation as a promising targeted drug delivery module.

\section{REFERENCES}

1. Madhav NVS, Saini A. niosomes: a novel drug delivery system, International Journal of research in pharmacy and chemistry, 2011; 1(3):498-511.

2. Sharma D, Ali AAE, Jayshree R. Aate, Niosomes as novel drug delivery systems: Review article. PharmaTutor, 2018; 6(3):5865. https://doi.org/10.29161/PT.v6.i3.2018.58

3. Seleci DA, Seleci M, Walter JG, Niosomes as nanoparticular drug carriers: fundamentals and recent application. Hindawi Publishing Corporation Journal of Nanomaterials, 2016; Article ID 7372306, 13 pages. https://doi.org/10.1155/2016/7372306

4. Durga Bhavani G, Veera Lakshmi P, Recent advances of non-ionic surfactants-based nano-vesicles (niosomes and proniosomes): a brief review of these in enhancing transdermal delivery of drug, Future Journal of Pharmaceutical Sciences, 2020; 6:1-18. https://doi.org/10.1186/s43094-020-00117-y

5. Yadhav JD, Kulkarni PR, Vaidhya KA, Niosomes a review. Journal of Pharmacy Research, 2011; 4(3):632-636.

6. Mujoriya R, Bodla RB, Dhamande K, Singh D, Patle L, Niosomal Drug Delivery System: The Magic Bullet, Journal of Applied Pharmaceutical Science, 2011; 01(09):20-23.

7. Mohamad Saimi N.I, Salim N, Ahmad N, Abdulmalek E, Abdul Rahman, M.B, Aerosolized Niosome Formulation Containing Gemcitabine and Cisplatin for Lung Cancer Treatment: Optimization, Characterization and In Vitro Evaluation. Pharmaceutics, 2021; 13:59. https://doi.org/10.3390/pharmaceutics13010059

8. Rani N, Rana R, Thakur R, Single S, Formulation and evaluation of atorvastatin calcium niosomes, international journal of science and research archive, 2021; 02(01):116-124. https://doi.org/10.30574/ijsra.2021.2.1.0028

9. Ali F, Jafar A, Majid S, Maryam G, Neda A, Ali N, Methylene blueloaded noisome: preparation, physicochemical characterization, and in vivo wound healing assessment, Drug Delivery and Translational Research, 2020; 10:1428-1441. https://doi.org/10.1007/s13346-020-00715-6

10. Jankie S, Johnson J, Adebayo AS, Pillai GK, Pereir LMP, Efficacy of Levofloxacin Loaded Nonionic Surfactant Vesicles (Niosomes) in a Model of Pseudomonas aeruginosa Infected Sprague Dawley Rats, Advances in Pharmacological and Pharmaceutical Sciences, V 2020; Article ID 8815969, 7. https://doi.org/10.1155/2020/8815969

11. Hajizadeh MR, Maleki H, Fahmidehkar MBMA. In vitro cytotoxicity assay of D-limonene niosomes: an efficient nanocarrier for enhancing solubility of plant-extracted agents, Research in Pharmaceutical Sciences, 2019; 14(5):448-45. https://doi.org/10.4103/1735-5362.268206

12. Sayeda Salma. S.A, Asha A N, V Kusum Devi, Formulation and evaluation of niosomes of mirtazapine for nasal delivery, Indo
American Journal of Pharmaceutical Research, 2019; 9(05):2011-2037.

13. Lu B, Huang Y, Chen Z, Ye J, Xu H, Chen W, Long X, Niosomal Nanocarriers for Enhanced Skin Delivery of Quercetin with Functions of Anti-Tyrosinase and Antioxidant Molecules, 2019; 24:2322. https://doi.org/10.3390/molecules24122322

14. Anindita De, Nagasamy Venkatesh, M Senthil, Bharat Kumar Reddy Sanapalli, R Shanmugham, and Veera Venkata Satyanarayana Reddy Karri, Smart niosomes of temozolomide for enhancement of brain targeting, Nano biomedicine, 2018; 5:1-11. https://doi.org/10.1177/1849543518805355

15. Pankaj, Satish Chander Arora and Rashmi Manchanda, Formulation, Evaluation and Optimization of Glipizide loaded Niosomes, International Journal of Chemtech Research, 2018; 11(09):08-14. https://doi.org/10.20902/IJCTR.2018.110902

16. Barani M, Mirzael M, Mahani T, Evaluation of carum loaded Nisomes on breast cancer cells: Physicochemical properties, in vitro cytotoxicity, flow cytometric, DNA Fragmentation and cell migration Assay, Scientific Reports, 2019; 9:7139. https://doi.org/10.1038/s41598-019-43755-w

17. Shaikh K, Pandhe P, Kale S, Bothiraja C, Development and Evaluation of a Novel Drug Delivery System for Albendazole, Indian Journal of Pharmaceutical Education and Research, 2018; 52(3):408-415. https://doi.org/10.5530/ijper.52.3.47

18. Leila Kanaani, Iraj javadi, Meysam Ebrahimifar, Hasan Ebrahimi shahmabadi, Azim Akbarzadeh Khiyavi, Torkan Mehrdib, Effects of Cisplatin-Loaded Niosomal Nanoparticles on BT-20 Human Breast Carcinoma Cells, Asian Pac J Cancer Prev, 2017; 18(2):365-368. https://doi.org/10.31557/apjcb.2017.2.2.27-29

19. Nagalakshmi. S, Kalaiaperumal K, Arul JM, Chaudhari PS, Pushpalatha HB, Shanmuganthan S, Fabrication and Characterization of Herbal Drug -Loaded Nonionic Surfactant Based Niosomal Topical Gel, J. Pharm. Sci. \& Re, 2016; 8(11):1271-1278.

20. Ying Qi Xu, Wen Rong Chen, Jonathan K. Tsosie, Xi Xie, Niosome encapsulation of curcumin: characterization and cytotoxic effect on ovarian cancer cells, Journal nanomaterials, 2016; Article ID: 6365295. https://doi.org/10.1155/2016/6365295

21. Dwivedi A, Mazumder A, du Plessis L, du Preez JL, Haynes RK, du Plessis J, "In vitro anti-cancer effects of artemisone nanovesicular formulations on melanoma cells," Nanomedicine: Nanotechnology, Biology, and Medicine.2015; 11(8):2041-2050. https://doi.org/10.1016/j.nano.2015.07.010

22. Goyal G, Garg T, Malik B, Chauhan G, Rath G, Goyal AK, Development and characterization of niosomal gel for topical delivery of benzoyl peroxide, Drug Delivery, 2013; 22(8):10271042. https://doi.org/10.3109/10717544.2013.855277

23. Punitha valli, M. Vignesh, Formulation of niosomal suspension with enhanced oral bioavailability of diclofenac sodium, Journal of Global Trends in Pharmaceutical Sciences,2012; 2(3):656671.

24. Kamboj S, Saini V, Bala S, Formulation and Characterization of Drug Loaded Nonionic Surfactant Vesicles (Niosomes) for Oral Bioavailability Enhancement, The Scientific World Journal, 2014, Article ID 959741. https://doi.org/10.1155/2014/959741

25. Wagh VD, Deshmukh OJ, Itraconazole Niosomes Drug Delivery System and Its Antimycotic Activity against Candida albicans, International Scholarly Research Network. 2012, Article ID 653465, 7 pages. https://doi.org/10.5402/2012/653465

26. Jankie S, Johnson J, Adebayo AS, Pillai GK, Pereira LMP, Efficacy of Levofloxacin Loaded Nonionic Surfactant Vesicles (Niosomes) in a Model of Pseudomonas aeruginosa Infected Sprague Dawley Rats, Advances in Pharmacological and Pharmaceutical Sciences, V 2020, Article ID 8815969, 7 pages. https://doi.org/10.1155/2020/8815969

27. Han W, Wang S, Liangetal R, Non-ionic surfactant vesicles simultaneously enhance antitumor activity and reduce the toxicity of cantharidin," International Journal of Nanomedicine, 2013; 8:2187-2196. https://doi.org/10.2147/IJN.S43568

28. Shaker D.S, Shaker M.A, Hanafy M.S, Cellular uptake, cytotoxicity and in-vivo evaluation of Tamoxifen citrate loaded niosomes. International Journal of Pharmaceutics, 2015; 493:285-294. https://doi.org/10.1016/j.ijpharm.2015.07.041

29. Tavano L, Muzzalupo R, Mauro L, Pellegrino M, Picci N, Transferrin-conjugated Pluronic niosomes as a new drug delivery system for anti-cancer therapy, Langmuir, 2013; 29(41):12638-12646. https://doi.org/10.1021/la4021383 